\title{
Un éclairage hypothétique sur certaines formes vocales traditionnelles : l'improvisation poétique en temps mesuré
}

\section{Marie-Hortense Lacroix}

\section{(2) OpenEdition \\ Journals}

Édition électronique

URL : http://journals.openedition.org/ethnomusicologie/420

ISSN : 2235-7688

\section{Éditeur}

ADEM - Ateliers d'ethnomusicologie

\section{Édition imprimée}

Date de publication : 1 janvier 2004

Pagination : 89-117

ISBN : 2-8257-0910-7

ISSN : $1662-372 X$

\section{Référence électronique}

Marie-Hortense Lacroix, « Un éclairage hypothétique sur certaines formes vocales traditionnelles l'improvisation poétique en temps mesuré », Cahiers d'ethnomusicologie [En ligne], 17 | 2004, mis en ligne le 13 janvier 2012, consulté le 20 avril 2019. URL : http://journals.openedition.org/

ethnomusicologie/420

Ce document a été généré automatiquement le 20 avril 2019.

Tous droits réservés 


\title{
Un éclairage hypothétique sur certaines formes vocales traditionnelles : l'improvisation poétique en temps mesuré
}

\author{
Marie-Hortense Lacroix
}

1 L'improvisation poétique chantée (IPC) semble occuper aujourd'hui, dans le panorama des poésies orales musicalisées d'Europe et du monde latin, une place déclinante quoiqu'encore bien représentée. En témoignent les « beaux restes» des formes médiévales de débats, de joutes et de tournois poétiques que constituent par exemple le chjama è respondi corse, la gara poetica sarde, le bertsolarisme basque, ainsi que les diverses formes ibéroaméricaines de "chant de défi» (cantos a desafío), de «controverses», "chants de poètes», etc. En témoigne également la pratique encore bien vivante, notamment dans le milieu rural du territoire ibéroaméricain où j'ai eu l'occasion de l'étudier d'un peu plus près, d'une improvisation poétique chantée plus modeste mais omniprésente, à usage plus intime et festif: courtes strophes de compliment, d'hommage, de provocation ludique, d'échange sur un sujet quelconque, improvisées au cours d'une fête, d'une sérénade, etc.

2 Pratique déclinante, pourtant, cela paraît certain, du moins en ce qui concerne ces formes d'IPC traditionnelles. Sans que l'on sache très bien si cela provient de l'irruption du support audiographique, de la domination grandissante de l'écrit ou du déclin du mode de vie agro-pastoral auquel elles sont le plus souvent attachées, il est clair que ces traditions d'IPC, dont plusieurs sources rapportent par exemple la présence dans diverses régions de France encore au début du XXe siècle, connaissent aujourd'hui un net recul.

Il peut donc paraître surprenant de supposer que, dans un passé pas si lointain, le monde occidental ait pu abriter des traditions d'IPC destinées à certains chants essentiellement fonctionnels, par exemple qu'il ait été courant d'improviser des strophes poétiques pour faire danser une assemblée de gens du peuple. Pourtant, nous disposons à ce sujet de témoignages précis, tels que celui-ci, extrait d'un passage de l'Illustre servante de 
Cervantès, où l'on verra comment un gentilhomme, déguisé en porteur d'eau, fait danser au son de sa voix la clientèle d'une auberge sur un contrapás, puis une chaconne, avant d'être lui-même interrompu par la sérénade d'un autre poète-paysan des environs (Cervantès 2002: 166-167; je ne cite ici que le début de la scène):

Lope jouait de la guitare de telle sorte qu'on disait qu'il la faisait parler. Les servantes lui demandèrent [...] de chanter quelque romance; il répondit que, pourvu qu'elles le dansassent à la façon dont on chante et dont on danse dans les comédies, il en chanterait un volontiers et que, pour ne pas se tromper, elles n'avaient qu'à faire ce qu'il leur dirait en chantant, et rien d'autre. [...] Lope se nettoya la poitrine en crachant à deux reprises; pendant ce laps de temps il pensa à ce qu'il allait dire et, comme il avait un esprit vif et délié, avec une heureuse facilité d'improvisation, il commença à chanter de la sorte:

Salga la hermosa Argüello, moza una vez, y no más, $y$ haciendo una reverencia, dé dos pasos hacia atrás.

De la mano la arrebate el que llaman Barrabás, andaluz mozo de mulas, canónigo del Compás.

De las dos mozas gallegas que en esta posada están, salga la más carigorda en cuerpo y sin devantal.

Engarráfela Torote, $y$ todos cuatro a la par, con mudanzas y meneos den principio a un contrapás ${ }^{1}$

Diverses sont les sources qui rapportent ce type de pratiques dans le monde hispanique; en 1854, l'érudit Basilio Sebastián Castellanos écrit encore, à propos des seguidillas:

Ce genre de poésie est tellement en usage chez les Espagnols des deux sexes, que tous y font figure de poètes, avec une telle adresse et une telle ingéniosité que la moindre gourgandine, en s'accompagnant d'un tambour de basque, et le dernier des chenapans à la guitare, peuvent rester des jours entiers à improviser des seguidillas; et il en va de telle sorte que dans les danses de ce type, presque toutes [les strophes] sont des adresses des chanteurs aux personnes qui dansent, ou au principal objet de la fête ou de la célébration [...] (Crivillé i Bargalló 1988: 217).

Certes, je n'ai pour l'instant jamais entendu de seguidillas réellement improvisées. Ceci, cependant, n'a rien de surprenant: toutes les seguidillas que j'ai pu apprécier in vivo avaient perdu leur véritable fonction de chansons à danser, puisque la demande n'émanait pas d'une assemblée d'auditeurs souhaitant danser et réclamant pour cela un support aux musiciens'; au contraire, la production musicale était offerte par un groupe de musiciens, que l'on qualifierait volontiers de «folklorique», souvent pour illustrer une chorégraphie reproduisant une danse un tantinet désuète. Or, ce procédé de mise en spectacle, qui s'articule autour de la présence d'un public non participant et requiert une certaine perfection d'apparence formelle, ajouté au fait que le genre n'est plus pratiqué de façon régulière et dans un cadre fonctionnel, ne s'oppose-t-il pas au principe même 
d'improvisation? La difficulté réside dans le fait que l'improvisation découle du contexte, et disparaît avec lui.

7 Pourtant, à l'écoute de certaines seguidillas, il me semble que l'on peut déceler des traces de cette longue pratique improvisatoire que je soupçonne.

\section{Une seguidilla canarienne}

Prenons le cas d'une seguidilla canarienne, en l'occurrence de cette forme que les Canariens appellent « seguidilla robada», du fait qu'un chanteur « vole» (robar = voler) l'air et le dernier vers du chanteur précédent pour dérouler sa strophe (Noda Gómez: 34), mais qui pourrait bien être aussi une déformation de l'expression plus ancienne et traditionnelle en Espagne de "seguidilla rodada» (rodar = rouler), qui décrit l'enchaînement des strophes entre elles. Nous considèrerons, à titre d'exemple, celle qui figure, sous le titre de "Seguidillas del salto», sur le CD Los Campesinos (Lanzarote), Isla con viento (1997), plage 8, seguidilla composée sur un modèle traditionnel.

9 Le principe en est simple, comme le montre la transcription partielle présentée en document 1 (parties chantées, et notation de la partie de timple ${ }^{3}$ dans les interludes instrumentaux, sur les trois premières strophes). Les chanteurs, ici au nombre de trois (A, $\mathrm{B}, \mathrm{C})$, chantent tour à tour une strophe " seguidillesque» ${ }^{4}$ sur un même patron musical (S); entre ces strophes, s'intercalent des épisodes de transition (T) comparables aux relais d'une course: le chanteur, en répétant ses deux derniers vers, «tend» comme un témoin, au chanteur suivant, son dernier vers sur la ligne mélodique qui accompagnait aussi son premier vers (m1); le chanteur suivant reprend ce dernier vers sur la même mélodie et enchaîne avec le premier vers de sa strophe ${ }^{5}$, toujours sur la même mélodie m1.

On peut donc résumer cette forme poético-musicale, fondamentalement strophique (aux variations prosodiques et stylistiques près), par le tableau suivant:

\begin{tabular}{|l|l|l|}
\hline Parties & Support poétique & Support mélodique \\
\hline Introduction instrumentale & & \\
\hline Strophes (S), $\mathrm{N}$ & $\mathrm{v} 1$ & $\mathrm{~m} 1$ \\
& $\mathrm{v} 2$ & I1 (instrumental) \\
& $\mathrm{v} 2$ & Instrumental) \\
& $\mathrm{v} 3$ & $\mathrm{v} 4$ \\
& $\mathrm{v} 3$ & $\mathrm{~m} 1$ \\
\hline Transitions (T), $\mathrm{x}(\mathrm{N}-1)$ & $\mathrm{v} 4$ & $\mathrm{~m} 1$ \\
\hline Conclusion instrumentale & & \\
\hline
\end{tabular}

11 Notons d'ores et déjà le caractère litanique que véhicule cette forme, visiblement faite pour se dérouler dans un temps étale et sur un grand nombre de strophes. Preuve en est, 
pour l'auditeur, l'aspect insolite de la fin du chant (fin de la dernière strophe), dépourvu de la transition qui formait aussi conclusion de la strophe précédente et donc quelque peu inabouti, d'un point de vue poétique (fin sur un avant-dernier vers de strophe) comme musical (ouverture mélodique vers $\mathrm{m} 1$, qui reste béante). Cette sorte d'« effet de bord» se trouve ici renforcée du fait du nombre réduit de strophes chantées $(N=5)$, que je reproduis ici:

\begin{tabular}{|l|l|}
\hline (chanteur A) & $\begin{array}{l}\text { Seguidillas me pides } \\
\text { yo te las canto, } \\
\text { cójelas al vuelo } \\
\text { pegando un salto. }\end{array}$ \\
\hline (chanteur B) & $\begin{array}{l}\text { Cuando saltas bailando } \\
\text { las seguidillas, } \\
\text { todos quedan mirando } \\
\text { tus pantorrillas. }\end{array}$ \\
\hline (chanteur C) & $\begin{array}{l}\text { Cada vez que tú bailas } \\
\text { se me encabrita } \\
\text { el corazón del pecho, } \\
\text { niña bonita. }\end{array}$ \\
\hline (chanteur B) & $\begin{array}{l}\text { Tienes una cintura } \\
\text { unas caderas } \\
\text { que embelesan bailando } \\
\text { siempre a cualquiera. }\end{array}$ \\
\hline (chanteur A) & $\begin{array}{l}\text { El timple y la guitarra } \\
\text { hacen compaña } \\
\text { pa' tocar seguidillas, } \\
\text { las que tú bailas. }\end{array}$ \\
\hline
\end{tabular}

Un autre paramètre me paraît révélateur: l'alternance des chanteurs, systématique dans ce genre poético-musical. Dans toutes les manifestations d'IPC auxquelles j'ai pu assister, notamment à Cuba, elle fait partie des règles implicites de l'exécution: j'ai entendu des poètes-chanteurs improviser parfois des heures durant, mais aucun soliloquer plus de trois minutes d'affilée ${ }^{7}$. Comme dans tant d'autres domaines qu'elle régit, l'improvisation est ici une œuvre de tissage, une construction collective qui naît et s'édifie de la rencontre et du dialogue entre plusieurs imaginations individuelles. A Cuba, ce principe d'alternance peut prendre la forme d'une joute, d'un tournoi, d'une ronde tour à tour, d'un échange pluridirectionnel en contexte amical, etc. L'alternance apporte bien sûr, notamment lorsqu'il y a présence d'un public extérieur, une dimension de jeu préthéâtral; mais elle participe aussi pleinement aux mécanismes qui rendent possible l'élaboration de l'œuvre improvisée: d'une part, en la nourrissant par la confrontation de subjectivités différentes et l'apport continuel d'idées nouvelles; d'autre part, en réalisant une certaine économie, de voix et surtout d'invention, qui permet à l'exécution de se prolonger pour accompagner le temps naturellement long du spectacle ou de la fête. 
Fig. 1 : Seguidilla " robada », lles Canaries (Lanzarote)

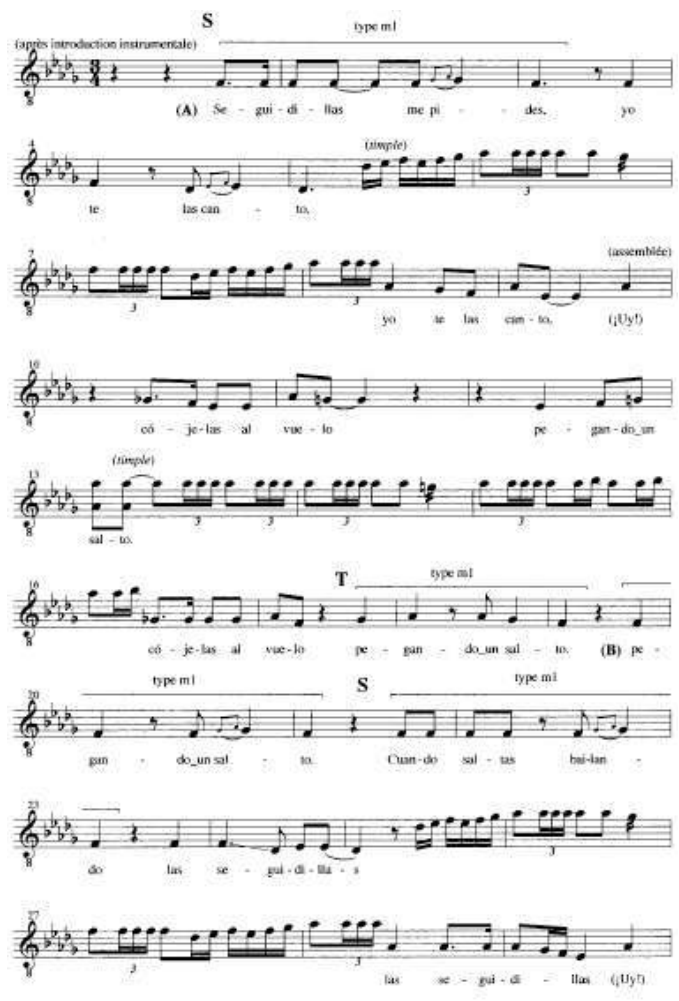

Fig. 1 (suite)
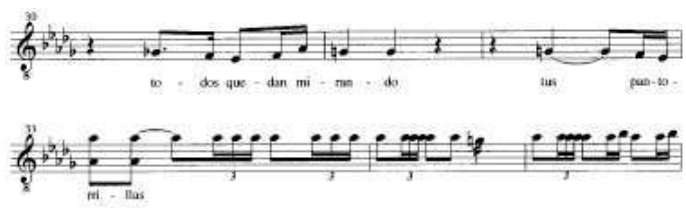

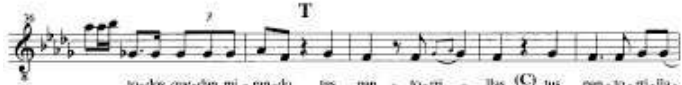
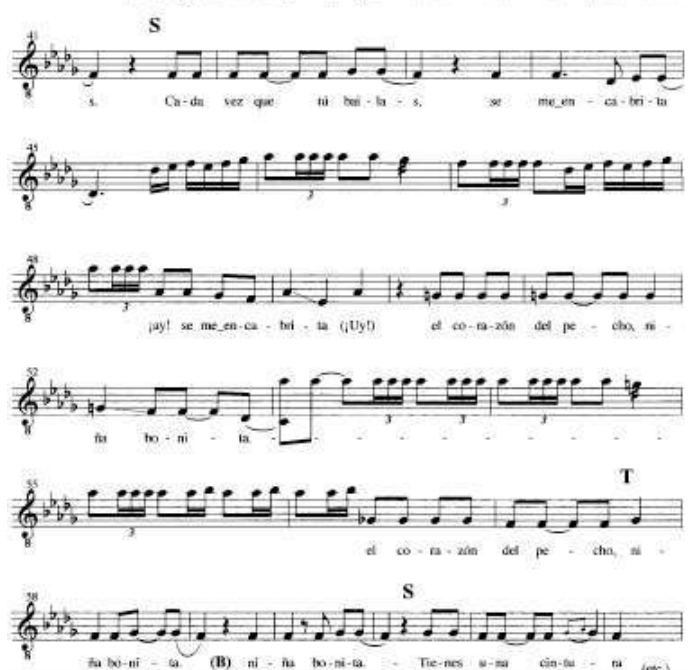

13 Ici, cette alternance prend le caractère particulier du tuilage, même si celui-ci ne se réalise que de façon différée dans le temps et non par superposition directe, puisqu'un 
chanteur empiète sur la strophe (dernier vers) et la musique (m1) du précédent pour lancer sa propre intervention. Ce principe de tuilage n'est pas sans évoquer le kan ha diskan breton, dont nous verrons plus loin qu'il peut constituer un éminent support d'IPC. On peut y percevoir une certaine dimension symbolique: deux chanteurs successifs se transmettent comme un flambeau le chant, le rôle de chanter et ici de mener la danse. Mais, dans le cadre d'une pratique improvisatoire, ils se transmettent aussi la responsabilité du devenir du poème. De même que dans ces contes collectifs où chaque participant prend en main, en parole, le conte au point où l'avait amené le conteur précédent pour le faire évoluer selon son inspiration, les chanteurs se transmettent ici le pouvoir de diriger la vie de la chanson.

14 Mais, outre ces similitudes formelles avec l'IPC, un autre type d'approche pourrait permettre d'examiner le caractère improvisatoire de cette forme poético-musicale.

On peut en effet, à l'instar des thermodynamiciens qui, pour examiner les conditions de réalisation d'une transformation physico-chimique, dressent un profil de l'énergie que demande chacune de ses étapes, analyser de même le processus d'improvisation d'une strophe dans la répartition de temps qu'impose la forme musicale (cf. fig. 2). Sont considérées ici, comme paramètres déterminants, les difficultés liées à la contrainte métrique (que l'on suppose proportionnelle au nombre de pieds du vers), aux contraintes prosodique et rimique, et à la contrainte temporelle (posée comme inversement proportionnelle au temps dont dispose le chanteur pour préparer son vers, compté ici en nombre de pulsations entre l'audition du dernier mot du chanteur précédent, et le début du vers en question). L'« énergie» que demande le processus n'est pas ici une quantité d'électron-Volts, mais bien cette énergie mentale particulière que requiert l'improvisation.

16 Une synthèse globale et qualitative révèle dans notre cas l'existence de deux «barrières» énergétiques: la principale pour le premier vers et une autre, moindre, pour les troisième et quatrième vers.

Il est alors probant d'examiner, comme le ferait tout bon thermodynamicien, les apports d'énergie que fournit la forme musicale au chanteur pour favoriser l'improvisation. Or, il me semble que cette énergie réside essentiellement dans la quantité de temps de préparation réellement efficace pour le chanteur, soit le temps de préparation durant lequel il n'a pas à chanter (interludes instrumentaux, répétitions finales du chanteur précédent) et peut pleinement réfléchir, « entendre» mentalement ce qu'il va chanter. Et l'on constate que cette « réponse» de la forme musicale à la contrainte improvisationnelle paraît ici tout à fait adaptée, puisque le chanteur dispose de 19 pulsations « silencieuses» avant de devoir entamer son premier vers, et de neuf autres avant le troisième. L'apport énergétique proposé par la forme musicale répond donc parfaitement aux besoins de l'improvisateur éventuel (cf. Fig. 2bis).

18 Mais finissons-en avec ce formalisme inhumain. Un chanteur n'est bien sûr pas un électron, et il faudrait prendre en compte bien d'autres paramètres liés à sa noble nature: stratégie, pouvoir de prévision, dons particuliers, culture en formules et «trucs» divers, etc. Cependant, ce type d'analyse permet de montrer le niveau d'adaptation de la forme poético-musicale même à un éventuel contexte improvisatoire, niveau d'adaptation qui peut être tel que l'on puisse supposer que tout un chacun, au sein d'une nation qui pratique couramment ce sport, puisse s'y adonner. Et donc, probablement, s'y soit adonné au temps où cette forme revêtait encore un caractère vivace et fonctionnel. 
Fig. 2 Profils « énergétiques » de processus d'improvisation d'une strophe (seguidilla robada)
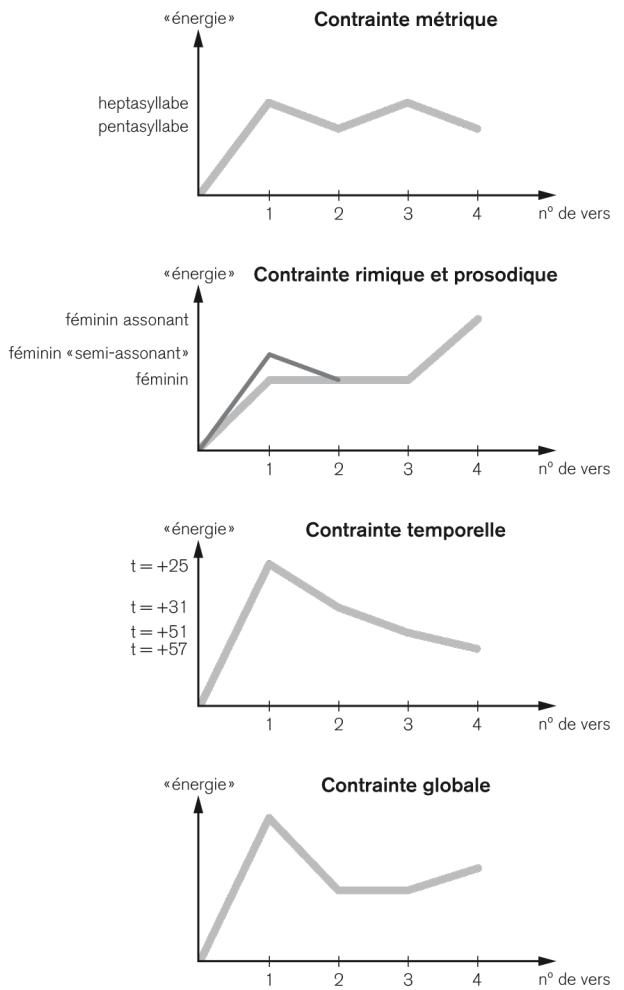

Fig.2bis: « Réponse» temporelle de la forme musicale à la contrainte improvisationnelle
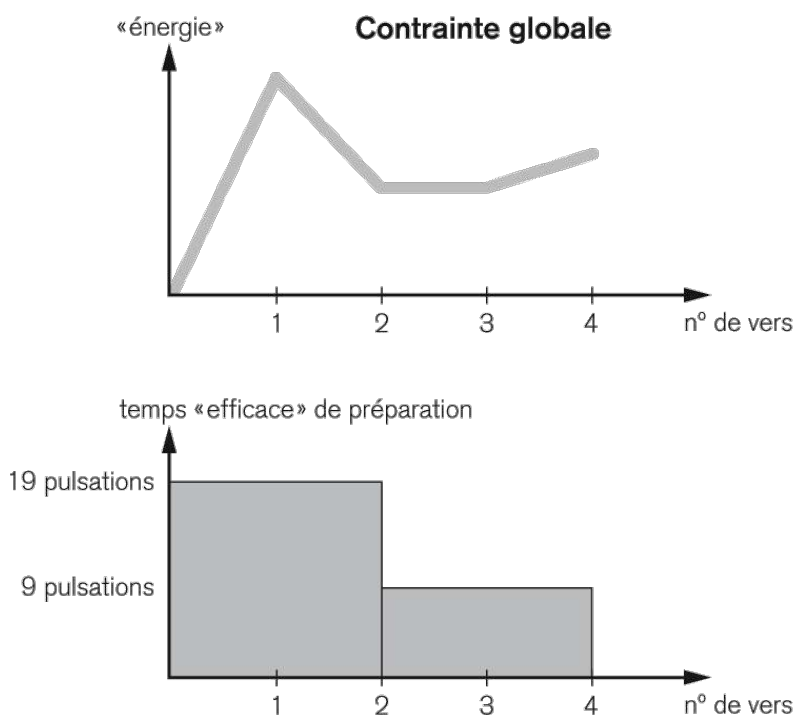

En outre, j'ai eu confirmation partielle de cette supposition. Différents informateurs canariens m'ont en effet affirmé que, il y a encore vingt ou trente ans et avant que ne se répande aux Iles Canaries la mode d'improviser sur des formes importées de Cuba, l'on improvisait volontiers sur à peu près toutes les formes de la chanson traditionnelle locale, «por isas, por folias, por seguidillas, etc.» D'ailleurs, n'ai-je pas maintes fois entendu, à Cuba, 
échanger des quatrains improvisés sur une musique de son, genre relativement figé qui sert de «bailable» (chanson à danser) dans les fêtes paysannes, alors que les canons officiels veulent que l'on improvise en décimas sur du punto?

De là à penser que ces formes musicales s'élaborèrent ou se transformèrent peu à peu en rapport avec les pratiques improvisatoires qu'elles accompagnaient, et qu'une bonne part de leur constitution formelle en découle, il n'y a qu'un tout petit pas.

Le choix de cette seguidilla canarienne procédait essentiellement d'une intuition, née de l'habitude de côtoyer des formes supports d'IPC, qui m'a conduite à en rapprocher cette forme poético-musicale aujourd'hui figée.

Depuis, cette intuition s'est souvent répétée, et il y aurait fort à parier que ce type d'analyse puisse donner des résultats intéressants sur de nombreuses formes vocales traditionnelles ${ }^{8}$. Si je ne craignais d'abuser, j'aurais donc bien d'autres exemples à détailler ici, notamment dans la sphère ibérique, mais aussi italo-tyrrhénienne (Italie, Sicile, Sardaigne, Corse), terres notables d'improvisateurs et d'improvisatrices. Dans ces dernières, en particulier, j'en suis arrivée à la conclusion que la plupart des chants monodiques à caractère originellement fonctionnel (berceuses, chants funèbres, chants d'opinions ou de lamentations, demandes et réponses, chants de travail, de tarantisme, de quêtes, chansons à danser, sérénades, contrasti, etc.) ont dû être longtemps pensés, et le sont peut-être encore aujourd'hui dans certaines contrées, comme indissociables d'une pratique improvisatoire.

\section{Sur les bords de la mer Tyrrhénienne}

23 Je ne puis détailler davantage dans le cadre de cet article et me permettrai simplement de citer quelques extraits d'ouvrages qui sont venus a posteriori confirmer cette intuition et mes analyses "thermodynamiques», par exemple dans le cas de la Corse. Ces ouvrages datent pour la plupart de la première moitié du $\mathrm{XX}^{\mathrm{e}}$ siècle, époque où l'on avait encore, semble-t-il, maintes occasions d'y entendre improviser des chants fonctionnels, notamment par des femmes et en particulier dans un contexte funèbre.

24 Austin De Croze, en 1911 (De Croze 1976: 34-35), écrit ainsi: «Les chants corses, nous l'avons dit, sont la plupart du temps improvisés, et improvisés par des montagnards, des paysans ou des bergers. [...] Les femmes sont également, et peut-être plus encore, douées d'inspiration. Toutes jeunes elles s'y exercent par des chants alternatifs ${ }^{9}$, sorte de pantoum rudimentaire, en vaquant aux soins du ménage et aux travaux champêtres. Il nous fut bien souvent donné de rencontrer telle de ces poétesses villageoises qui, à notre entrée dans la maison, la casa, et après que l'hôte nous avait souhaité la bienvenue, se levait, nous présentait une fleur, un verre d'eau ou une poignée de châtaignes et nous adressait une strophe de circonstance.»

Et, citant le témoignage de Paul Bourde: «Je ne crois pas qu'il y ait un autre peuple en Europe chez lequel la pensée s'exprime ainsi spontanément dans des formes rythmées. Presque tout le monde est capable de faire de ces chants, plus d'un bandit se distrait de ses longues stations dans le maquis en rimant un récit de ses malheurs ${ }^{10}$.»

Il ajoute, ébauchant la réflexion à laquelle nous nous livrons en ce moment (De Croze 1976: 38-39): «Cette rime qui, dans les chants corses, revient alternativement trois fois, serait en réalité le temps fort du rythme musical moderne appliqué au rythme poétique et facilitant, par cela même, l'improvisation ${ }^{11}$.» 

Colucci 1996: 8-15): «La nouvelle génération, par la force des choses, s'éloigne des vieux usages. Le dolu n'a plus dans de nombreux villages sa valeur primitive; les voceratrici, les préfiques inspirées sont appelées de plus en plus rarement pour improviser autour de la tola $^{12}$. [...] Toutefois, rares sont les villages qui n'ont plus leurs improvisatrices et de nombreuses personnes des deux sexes, capables de réciter de longues heures voceri, lamenti, sérénades anciennes et modernes, paghjelle [...].»

"Le vocero (vocerato, ballata), improvisé invariablement par les femmes (le nom change selon la localité), est l'expression la plus pure de la poésie corse primitive et la plus noble manifestation de son art populaire. Au centre et au sud de l'île, la mort est encore entourée des cérémonies des anciens temps.»

Suit une description de ces cérémonies funèbres: « [...] Brusquement, une femme se lève. Se penchant au-dessus du mort, celle-ci commence sa ballata, pendant qu'elle se balance de manière rythmique comme si elle berçait celui qu'elle pleure. Sa voix ne s'élève jamais au-dessus du murmure monotone de la mère qui endort son enfant. Les strophes cuivrées par de longues notes étirées se suivent sans interruption en grave cantilène, comme une leçon apprise par cœur. La voix court sans intervalle, d'une note mineure à l'autre (sic), se tenant toujours dans la brève gamme de la musique montagnarde. [...] le chant semble ne pas vouloir cesser, quand une autre silhouette se lève silencieusement et commence à improviser. [...] La caracole, la macabre ronde des préfiques autour du cercueil a désormais disparu, ou presque. Il n'y a pas si longtemps, on la dansait encore dans les villages reculés.»

Cette description d'une forme poético-musicale, toute ingénue qu'elle soit, évoque bien d'autres formes de chant monodique en usage, notamment, en Sardaigne et en Corse. L'auteur ajoute d'ailleurs: « Dans les endroits où les usages primitifs commencent à être abandonnés, si les femmes ne sont plus appelées à improviser pour les morts, elles le font pour les vivants. Elles ont la veine créatrice facile pour chaque événement familial et on leur doit les délicieuses ninne-nanne, pour bercer les enfants. Les lamenti d'amour pour le fiancé sous les drapeaux, les fiori ou les sermoni de mariage, et les chansons pour les élections. [...] Ce sont habituellement les hommes qui composent les chansons humoristiques plus ou moins salaces qui font les délices des veillées autour du fugone, et ces modestes poètes montagnards, qui savent improviser entre eux des chjama-rispondi durant des heures entières, sont des auteurs de brindisi et de contrasti héroïco-comiques à chanter en solo ou en paghjella, c'est-à-dire avec certaines strophes répétées en chœur.»

Ghjermana De Zerbi, en 1981 (1981 : 14-15), nous précise quant à elle que le terme versu désigne à la fois la forme de la strophe et la mélodie qui lui sert de vecteur; ligne mélodique dont l'extrême simplicité s'explique par le souci de mettre en valeur les paroles et de pouvoir les mémoriser dans l'instant [et pourquoi pas, les improviser, nda].

L'auteur distingue ainsi différents types de versu, selon la fonction à laquelle ils sont destinés, et qui se déclinent en plusieurs modèles (versu currente ou versu di l'improvisatori pour le chjama è respondi et la serenata, versu di i lamenti, versu di e canzone d'elezzioni, versu di e nanne, versu di i voceri), tout en concédant qu'il existe des analogies de structure et un certain niveau de mélanges entre ces catégories. Elle semble donc considérer, en accord avec ce que rapportent une majorité d'observateurs actuels, l'improvisation poétique chantée corse comme relativement confinée au chjama è respondi, voire au contrastu, et à la serenata, alors que les auteurs plus anciens l'étendent à divers genres vocaux monodiques

Cahiers d'ethnomusicologie, 17 | 2004 
et fonctionnels (nanna, lamentu et vocero en particulier). La situation semble donc avoir changé entre l'avant-guerre et 1981. Toutefois, on peut supposer que cette façon particulière de penser le chant, liée à un caractère improvisatoire d'origine, s'est transmise à sa forme poético-musicale et y reste attachée.

Il n'est, certes, jamais clairement dit que tous les genres de la chanson fonctionnelle, monodique ou en paghjella, aient ressorti d'une pensée improvisatoire. Cependant, les analogies et les équivalences qui existent entre les formes vocales attestées ou non comme improvisatoires - ne serait-ce que la relative interchangeabilité et les fortes ressemblances qui lient différents types de versi -, laissent peu de doutes quant au fait que certaines auraient pu inexplicablement déroger à cette conception générale de la chanson.

Fig. 3: L'apprentissage de l'improvisation au berceau (Cuba, Sancti Spíritus)

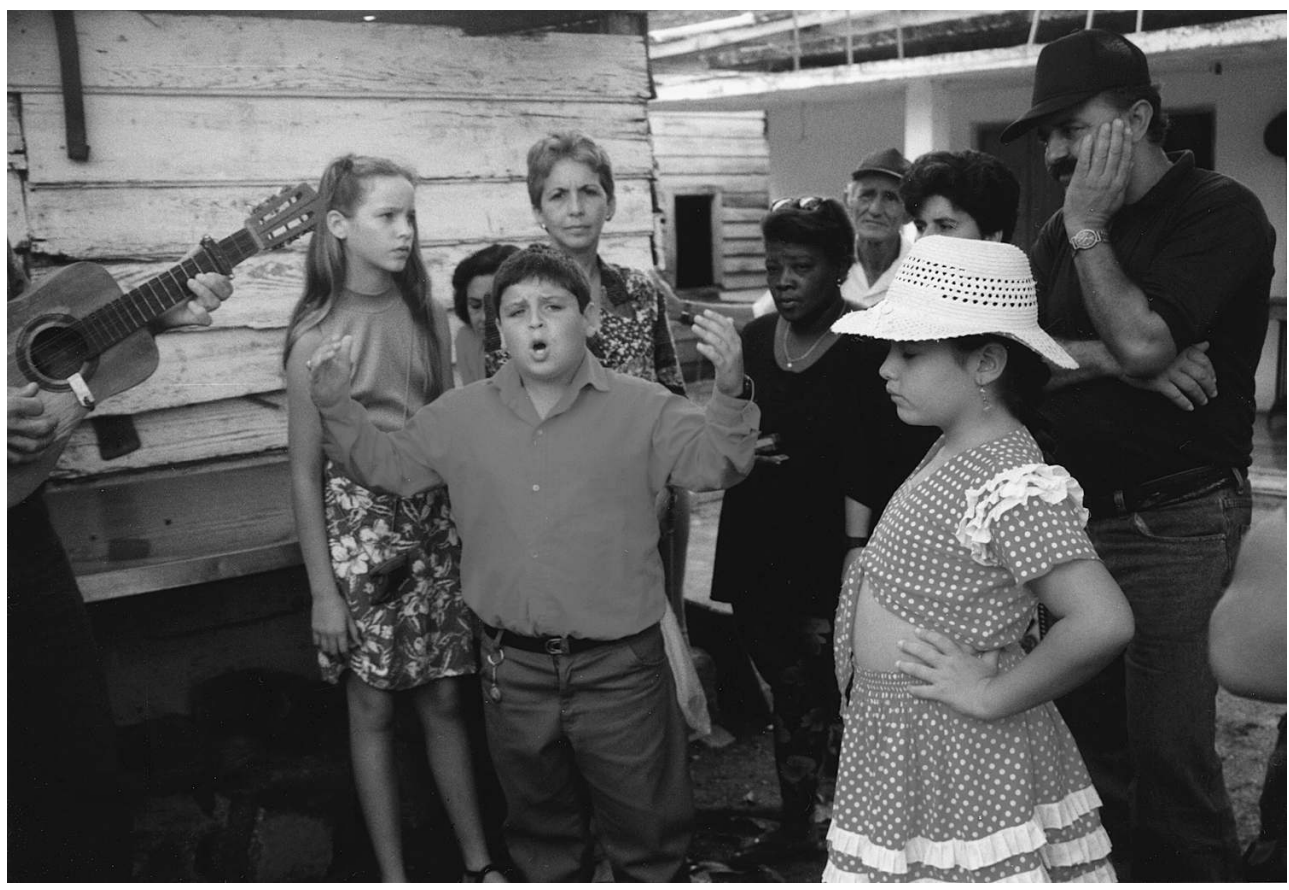

\section{Et chez les francophones?}

Parmi les caractéristiques formelles qui, dans le domaine corse, évoquent nettement une conduite improvisatoire à l'origine, il serait intéressant d'en citer quelques unes qui se trouvent également dans les répertoires improvisés ibéroaméricains, ou que nous retrouverons dans le domaine de la chanson traditionnelle française, à commencer par la structuration poétique en vers longs assonancés et fortement césurés (typiquement, le terzinu corse $\left.{ }^{13}\right)$.

Cependant, deux différences fondamentales séparent le domaine italo-tyrrhénien et le domaine ibérique du domaine français: l'importance de répertoires à voix nue de type non ou faiblement mesuré, i.e. non soumis à un temps rigide, inélastique, et la conduite partiellement mélismatique de la voix. On comprendra aisément que ces modalités du chant, auxquelles obéissent la plupart des répertoires dédiés à l'improvisation poétique "pure», soient les plus propices à l'exercice improvisatoire: le poète qui chante à voix 
nue, seul et non asservi au joug d'une structure temporelle imposée, adapte de lui-même son débit verbal, la longueur de ses silences, éventuellement l'utilisation de répétitions textuelles, aux difficultés que lui pose l'improvisation; le développement mélismatique de certaines syllabes, outre le temps supplémentaire qu'il lui donne de façon stratégique, lui permet aussi d'«absorber» d'éventuelles irrégularités métriques ${ }^{14}$. Dans ces répertoires, la forme musicale, organisme mouvant qui se construit et s'adapte au fur et à mesure, semble posséder un rôle à la fois illustratif et fonctionnel: par son caractère psalmodique ou son profil inexorablement descendant ${ }^{15}$, la mélodie vocale paraît reproduire - et, par synergie, soutenir - le souffle de l'inspiration poétique, que celui-ci s'incarne en une longue litanie (dans les berceuses, les lamentations funèbres, les chants de travail, de marche, etc.) ou en «clameurs» successives (dans les joutes et chants de défi, les complaintes, etc.).

En revanche, dans la chanson traditionnelle française comme dans tous les répertoires vocaux mesurés, i.e. soumis à ce temps « inélastique» qui est une perpétuelle contrainte pour l'improvisateur - répertoires qui ne peuvent souvent donner lieu qu'à une pratique semi-improvisatoire ${ }^{16}$-, c'est avant tout la forme poético-musicale, fixe, qui doit présenter des réponses fonctionnelles à la contrainte d'invention poétique: schéma d'assonances et de répétitions textuelles adapté, utilisation de refrains plus ou moins onomatopéïques, de formules ou de membres de phrase dénués de sens, construction par collage de séquences, stratégies de gain de temps faisant intervenir des ritournelles instrumentales, des répétitions responsoriales (ce qui fut peut-être le rôle originel de la paghjella corse ?), etc.

37 Si surprenant que cela puisse paraître, il est ainsi possible de discerner une excellente synthèse de ces « réponses fonctionnelles» de la forme à l'improvisation, dans le domaine de la chanson traditionnelle française.

Rappelons-nous d'abord que celle-ci, étant fortement caractérisée par une conduite syllabique de la mélodie vocale, se trouve, comme aucune autre peut-être, assujettie en un rapport «inélastique» au temps imposé par le déroulement du texte (Laforte 1997: 20-21).

D'autre part, le caractère fonctionnel, à l'origine, de bon nombre de ces chansons les a très souvent soumises à un carcan rythmique précis et peu enclin à la variation. Penchons-nous par exemple sur le cas très répandu de ce que Conrad Laforte décrit comme la "chanson en laisse», i.e. reposant sur la succession d'un nombre variable de vers isométriques et uniformément assonancés, forme métrique qu'il considère comme directement héritée de la laisse médiévale ( $c f$. exemple présenté en fig. 4). Cet ensemble de formes poético-musicales revêt, dans la tradition orale française, un fort caractère fonctionnel, puisqu'il servait essentiellement à accompagner la marche, des danses en rond ou des travaux collectifs. A la tutelle temporelle du texte, se superpose donc celle de la structure rythmique imposée par la danse, la marche ou le travail. Il y a donc là un exemple parfait de ce temps rigide auquel doit se soumettre le chanteur et qui, dans l'hypothèse d'une conduite improvisatoire ou semi-improvisatoire des paroles, lui interdit la moindre hésitation, le moindre «temps mort». D’où la nécessité absolue d'avoir recours à diverses stratégies visant à assouplir les contraintes de l'improvisation poétique: gestion appropriée du temps de préparation, facilité assonantique, souplesse du rapport mémorisé/improvisé, existence de substituts de la parole prêts à l'emploi, etc. 
Fig. 4: « Le Galant oublié dans l'armoire» (d'après Conrad Laforte)

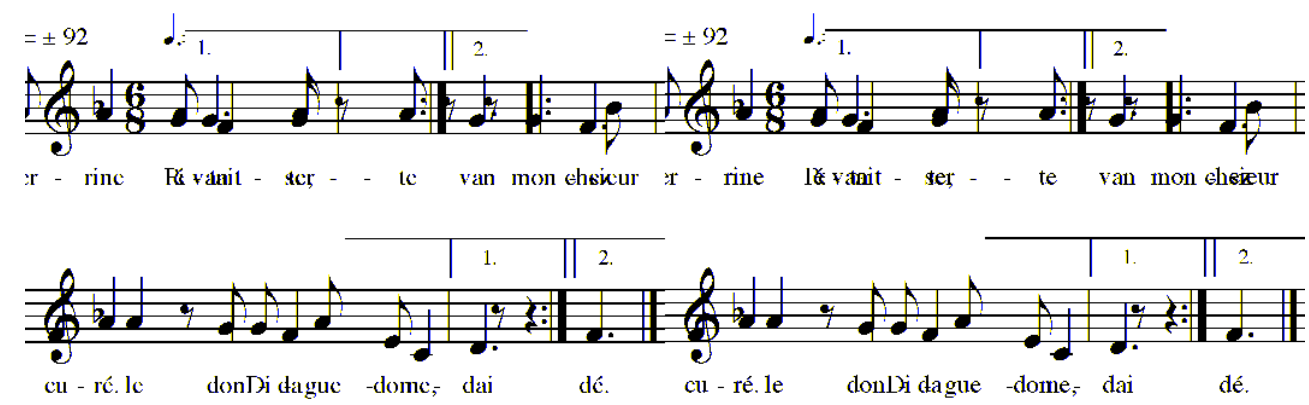

Perrine était servante (bis) chez monsieur le curé.

Digue donda dondaine

chez monsieur le curé.

Digue donda dondé

Son amant viens la voir-e un soir après l' dîner.

- Perrine oh! ma Perrine voudrais-tu m'embrasser?

- Et grand nigaud qu' t'es bête ça s' prend sans se demander.

- V'là m'sieur l' curé qu'arrive où j'vas t'y ben t' cacher.

Cache toi donc dans la hûche Il n' saura point t' trouver.

Au bout de six semaines les rats l'avaient bouffé.

On fit creuser son crâne pour faire un bénitier.

On fit monter ses jambes pour faire un chandelier.

Voilà la trist' histoire d'un jeun' homm' à marier.

Qu'allait trop voir les filles un soir après dîner.

Or, nous allons voir que plusieurs schémas formels couramment utilisés par la chanson traditionnelle française peuvent n'apparaître que comme les outils privilégiés de cette stratégie.

Prenons, à titre illustratif, un exemple. La fig. 4 présente la chanson dite du «Galant oublié dans l'armoire» telle que la retranscrit Conrad Laforte (1997: 654-655), et dont nous apprenons que, répandue notamment dans l'Ouest français, elle accompagnait des rondes à danser. Cette chanson combine, au sein d'une structure poético-musicale composite, plusieurs principes formels typiques de la chanson traditionnelle française en temps mesuré: forme en laisse, forme ternarisée de type $\mathrm{ABB}$, formule strophique dite du « rabéraa», forme à répons et forme à refrain plus ou moins onomatopéíque (dite «tralala»); principes formels dont nous allons essayer de montrer que leur constitution comme leur agencement contribuent à engendrer une forme poético-musicale «à faible demande énergétique», propice à une improvisation continue.

\section{La forme en laisse}

Comme nous l'avons vu et comme le montre la présentation adoptée par Conrad Laforte dans la fig. 4, la chanson en laisse s'articule autour d'une suite continue de vers isométriques uniformément assonancés (ici, en -é), vers le plus souvent longs et munis d'une forte césure, laquelle respecte le principe d'alternance entre désinences féminine (ici à la césure: « servante») et masculine (ici à l'assonance: -é). Cette laisse constitue une trame narrative dont chaque vers (éventuellement chaque couple de vers) se voit développé au sein d'une formule strophique donnée, formule qui souvent marque la césure au point que l'on perçoit le vers long comme un distique assonancé au vers pair. 

type de vers dont nous avons déjà constaté avec Pierre Bec qu'il était extrêmement courant dans les répertoires sujets à l'IPC, ce qui se conçoit par ailleurs en termes d'aisance improvisatoire: pour une quantité de paroles correspondant à un distique, l'improvisateur n'est contraint de fournir qu'une simple assonance, a fortiori le plus souvent choisie parmi les plus « faciles» qui soient (-é, -age, -an, etc.). Comme le montre le deuxième vers de notre exemple («Son amant vint la voir-e...»), il n'a en outre pas tellement à s'inquiéter de la réelle féminité de sa césure. La contrainte assonantique, bien qu'assez réelle, n'est d'ailleurs pas non plus absolue, comme le montrent diverses irrégularités demeurées dans la tradition ( $c f$. par exemple Malbrough s'en va t'en guerre $)^{17}$. La contrainte métrique, enfin, est des plus faibles, puisque le « distique» en question se compose de vers courts, typiquement d'hexasyllabes ${ }^{18}$, dont on pourrait montrer qu'ils sont aussi naturels et propices à l'improvisation, pour un francophone, que peuvent l'être par exemple les octosyllabes pour un latin.

Par ailleurs, l'extrême simplicité structurelle de la laisse affranchit l'improvisateur potentiel d'une dernière contrainte, d'ordre strophique: improviser une strophe nécessite que l'on ait toujours en mémoire le schéma rimique à suivre et l'endroit où l'on se situe pour savoir la rime que devra respecter le vers que l'on va chanter ${ }^{19}$; pour improviser une laisse, il suffit de se souvenir, localement, d'une seule assonance à respecter.

Une autre particularité de la laisse, susceptible de la rendre propre à l'improvisation poétique, réside dans son degré de souplesse et d'ouverture: de même que, dans les rondes et farandoles qu'accompagnent ces chansons, un quidam peut à tout moment s'insérer dans la chaîne des danseurs, la quitter ou la prolonger, de même, la forme de la laisse - une chaîne de vers reliés par une assonance commune, et dont le nombre peut varier à l'envi - permet à chaque instant au chanteur d'intercaler, d'ajouter, d'ôter ou de remplacer un vers ou un groupe de vers donné. Et ce d'autant plus que, comme le rappelle Conrad Laforte (1981: 21-23), chaque vers de laisse constitue une unité syntaxique, autonome du point de vue formel.

Notons par ailleurs que bon nombre de chansons en laisse réduisent au minimum (typiquement, par un procédé proche de l'énumération, à un mot) la part d'innovation poétique de chaque vers, en reprenant une grande partie du vers comme matrice du suivant, comme on peut le constater par exemple ici:

Fig. 5

La laine des moutons

C'est nous qui la tondaine

La laine des moutons

C'est nous qui la tondons

Tondons, tondons,

La laine des moutaine (s? ?)

Tondons, tondons

La laine des moutons

La laine des moutons, c'est nous qui la lavons

La laine des moutons, c'est nous qui la cardons

La laine des moutons, c'est nous qui la filons

[...]

$\mathrm{Au}$ niveau historique, rappelons que la laisse servait à l'origine aux chansons de geste médiévales, poèmes épiques de plusieurs milliers de vers dont l'interprétation orale de 
longs extraits nécessitait forcément une part d'improvisation. Mais on peut très bien imaginer que le mode du récit, souvent plus proche de la parole et non soumis à un rythme mesuré, laissait à l'exécutant le loisir d'improviser et d'enchaîner les vers de laisse à son gré, ce qui peut expliquer que les chansons de geste se présentent à l'écrit comme des séries de laisses énoncées telles quelles, où les vers «nus» (comme des vers) s'enchaînent les uns aux autres. En revanche, le contexte continûment musicalisé et mesuré de la chanson fonctionnelle impose que l'improvisation se fasse dans un temps donné et sans hésitation. Il faut donc entourer le vers d'une structure «temporisante» qui rendra plus aisée et plus sûre son improvisation.

Dès lors, il y a lieu de se pencher plus précisément sur le rôle stratégique de la formule strophique qui «emmaillotte» chaque vers de la laisse. Ici, interviennent donc d'autres principes formels en usage dans la chanson traditionnelle.

\section{Le « rabé-raa»}

49 C'est par cette appellation aux consonances vaguement néanderthaliennes que le métricien Benoît de Cornulier (Cornulier 1996: 309-337) désigne une formule strophique des plus répandues dans la chanson traditionnelle française et dans la chanson en laisse en particulier. Cette formule se présente comme un quatrain composé de deux distiques de vers non forcément isométriques ( $c f$. fig. 6) qui suivent un schéma assonantique ab-aa («abé-aa») $)^{20}$ et dont le troisième répète textuellement le premier (d'où «ra»bé-«ra»a). L'exemple suivant, dont on voudra bien pardonner le caractère potache, en montre une structuration possible parmi des centaines.

Fig. 6

Arrêtez, arrêtez cocher,

J'ai trois poils du cul pris dans la portière,

Arrêtez, arrêtez cocher,

J'ai trois poils du cul pris dans l'marchepied.

Faut-il pour trois poils du cul, pour trois poils du con

Faire tant de manières

Faut-il pour trois poils du cul, pour trois poils du con

Faire tant de façons

$[\ldots]^{21}$

Notons que la figure 5 utilise également ce type de formule. Dans Le Galant..., le rabé-raa se trouve intégré dans la seconde partie de la strophe où il développe, moyennant l'usage de refrains «tralaliques», le second hémistiche du vers de laisse:

Chez monsieur le curé,

Diguedondadondaine,

Chez monsieur le curé,

Diguedondadondé.

51 Bien des raisons doivent pouvoir expliquer la formation et la fréquence d'emploi de ce type de formule dans la chanson traditionnelle: aspects illustratifs, correspondances chorégraphiques (notamment dans les danses en rond), mémorisabilité, intérêt ludique, etc. Et parmi elles, doit figurer en bonne place son adéquation à une pratique improvisatoire.

Dans cette perspective, la première vertu du rabé-raa pourrait être de donner du temps, moyennant une stratégie inventive adaptée. D'une façon générale, il s'agit d'énoncer un 
quatrain en n'inventant qu'un distique, un vers, voire un simple mot. Dans les cas les plus compliqués, comme la figure 6 , la stratégie consistera par exemple à trouver un distique («Arrêtez, arrêtez, cocher, / J'ai trois poils du cul pris dans l'marchepied»), puis un substitut féminin du dernier mot («la portière» = «l'marchepied»). Dans les cas les plus simples, comme la figure 5 , un simple verbe disyllabique suffira à alimenter deux quatrains successifs: on a donc réduit au maximum le rapport quantité d'innovation poétique / temps d'énonciation, et donc la difficulté de l'improvisation.

Une seconde vertu improvisatoire, qui concerne cette forme poétique autant que sa musicalisation, pourrait résider dans la théâtralité qu'elle sous-tend. Comme le rappelle Benoît de Cornulier dans l'article cité, le sens des deux distiques du rabé-raa correspond en général à un « contraste final sur fond de répétition initiale», doublé d'une opposition " inachevé/achevé» ${ }^{22}$ que souligne l'alternance d'une rime finale féminine (b) et d'une rime finale masculine (a). Musicalement, ceci se traduit par une parfaite structure phraséologique de type "antécédent/conséquent», où une même phrase mélodique ( $\mathrm{m} 1$, accompagnant le vers "ra») s'enchaîne à une phrase suspensive ${ }^{23}$ dans le premier distique ( $\mathrm{m} 2$, sur le vers «bé»), et à une phrase apparentée mais conclusive dans le second (m2', sur le vers « a»).

Fig. 7: Structuration mélodique en « antécédent/conséquent» du rabé-raa

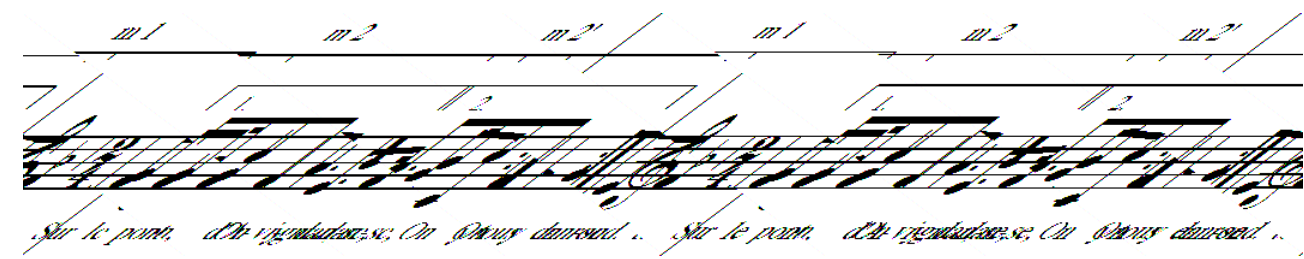

On peut donc bien imaginer le côté piquant que permet d'introduire une telle forme poético-musicale lorsque les paroles en sont improvisées, et que le public est en attente du sel qu'apportera le dernier mot, ou qu'au contraire il le sait ou le devine dès le premier vers... ${ }^{24}$

\section{La forme ternarisée $A B B$, la forme à répons, la forme à refrain («tralala»)}

Par «forme ternarisée», j'entends la catégorie de formules - fréquemment relevée en Bretagne et dans l'Ouest français, mais aussi présente sur le reste du territoire (Rivière 1991: 234-235) - qui tend, en répétant le deuxième vers d'un distique (ou hémistiche d'un vers de laisse), à conférer à la strophe poético-musicale un aspect ternaire de type $\mathrm{ABB}$.

Dans Le Galant..., par exemple, cette forme se combine avec le rabé-raa pour structurer globalement la strophe sur:

Perrine était servante

Chez monsieur le curé

Chez monsieur le curé

57 Ici, elle se mêle d'autre part à une forme à répons (dans la pratique traditionnelle, l'assemblée répète en chœur le premier vers: « Perrine était servante»...) et à une forme à refrain plus ou moins onomatopéique, de type dit "tralala» (Diguedondadondaine, Diguedondadondé). Il est bien évident que ces divers procédés visent, encore une fois, à "gagner» du temps ou à « économiser» du texte en diminuant le rapport texte/temps ${ }^{25}$ : 
on pourrait déterminer une équivalence entre les différentes formes de temps neutre, vide du point de vue de l'innovation poétique, que constituent les répétitions ou répons, les refrains, tralalas ou paroles non signifiantes, ou encore les interludes instrumentaux. Or, ce temps gagné est potentiellement du temps de préparation, de l'énergie improvisatoire.

Dans le cas du Galant, la structure poético-musicale est trop simple pour qu'une analyse de type «thermodynamique» soit vraiment utile. On pourra cependant observer, qualitativement, que le temps de préparation est réparti de la manière suivante dans la strophe (d'après la transcription de la Fig. 4):

Perrine était servante

4 pulsations silencieuses (répons)

Chez monsieur le curé

12 pulsations chantées (répétition, tralalas)

Il ressort alors que le temps efficace de préparation est réparti de façon à peu près équivalente entre les deux hémistiches ${ }^{26}$, et d'une nature propice à l'effort demandé: un court temps silencieux pour «entendre» un hémistiche assonant, un long temps chanté pour réfléchir à l'idée du vers de laisse suivant et à un premier hémistiche à la forme peu exigeante ${ }^{27}$.

On voit à travers ce simple exemple, où divers principes formels se trouvent combinés en un tout organique et original ${ }^{28}$, comment ces principes formels et leur combinaison peuvent soutenir une pratique improvisatoire des paroles.

61 L'ensemble des répertoires de la chanson traditionnelle en France porte bien d'autres marques de cette pratique supposée: l'alternance des chanteurs (que l'on trouve notamment dans les différentes formes de chanson à répondre, et en particulier dans la modalité en «tuilage» du kan ha diskan de Bretagne intérieure), l'interchangeabilité des strophes et des supports musicaux pourvu qu'ils soient isomorphes ${ }^{29}$, le caractère formulaire de l'expression et l'utilisation de nombreux stéréotypes au niveau du contenu, les constructions par collages de citations (souvent extraites d'autres chansons, procédé que Conrad Laforte qualifie de "greffe»), l'existence d'articulations et de canevas types dans les structures narratives ${ }^{30}$, etc., éléments qu'il serait trop long de détailler ici mais qui sont en rapport direct avec notre hypothèse.

Par ailleurs, nous pouvons invoquer d'autres arguments qui viennent la corroborer. En premier lieu, divers témoignages qui montrent que, comme dans les villages canariens ou corses, l'improvisation poétique chantée "fonctionnelle» s'est pratiquée, voire se pratique encore, dans de nombreuses campagnes de France. Dans certaines régions de Bretagne, comme nous venons de le voir, elle fait encore l'objet de genres définis et de pratiques bien vivantes; Jean-Michel Guilcher évoque par exemple celle du galv, prélude à la danse qui peut comporter des plaisanteries improvisées destinées à l'assistance, de même que les formules versifiées par lesquelles les chanteurs terminent la danse, improvisations qui reposent encore une fois sur des vers longs assonancés et césurés ${ }^{31}$. Jean-Pierre Bertrand m'a récemment parlé des improvisations des anciens « darioleurs», meneurs de bœufs vendéens, qui accompagnaient et facilitaient leur labeur, ou encore du «boulité» dansé (cf. ci-après). Plusieurs gens de lettres du passé, notamment parmi les romantiques, ont fait allusion à de semblables pratiques, sans toutefois, malheureusement, retranscrire les vers patois dans lesquels elles s'exprimaient; on peut regretter par exemple que le témoignage de George Sand, dans l'appendice de La Mare au Diable, ne soit pas plus circonstancié. Cependant, quelques auteurs ont osé se donner la 
peine de ce précieux réalisme, en particulier au début du $\mathrm{XX}^{\mathrm{e}}$ siècle, que ce soit dans la littérature ou dans des ouvrages traitant de chanson traditionnelle. Ainsi, par exemple, nous pouvons lire dans La Table-aux-Crevés, de Marcel Aymé, la transcription d'une «longue litanie improvisée en patois de Cessigney» (près de Dole, Jura), en couplets de vers courts assonancés, une prière à la Vierge que chante Jeanne, inspirée par le vin d'Arbois, pour se libérer des affres de sa situation:

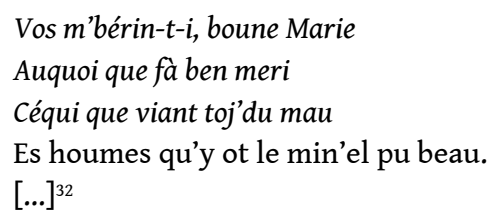

Joseph Canteloube décrit, de façon peut-être un peu romancée, certains chants improvisés de Haute-Auvergne comme les baylèros, chants de bergers (et bergères) échangés d'un sommet à l'autre: "Le dialogue est souvent improvisé sur les notes invariables de la mélodie: tantôt c'est un chapelet d'injures, tantôt des plaisanteries, tantôt un dialogue amoureux [...]» (Canteloube 1951). Louis Lambert, en 1906 (Lambert 1983: 3-7 et 344-345), rapporte l'accompagnement semi-improvisatoire des danses languedociennes menées au chant, en des couplets souvent humoristiques ou satiriques ${ }^{33}$. Il évoque également l'usage de la sérénade, alors encore en vigueur en Languedoc (Lambert 1983: 184).

Fig. 8 : Épisode du « boulité» dans un bal vendéen mené «à la goule» ${ }^{34}$

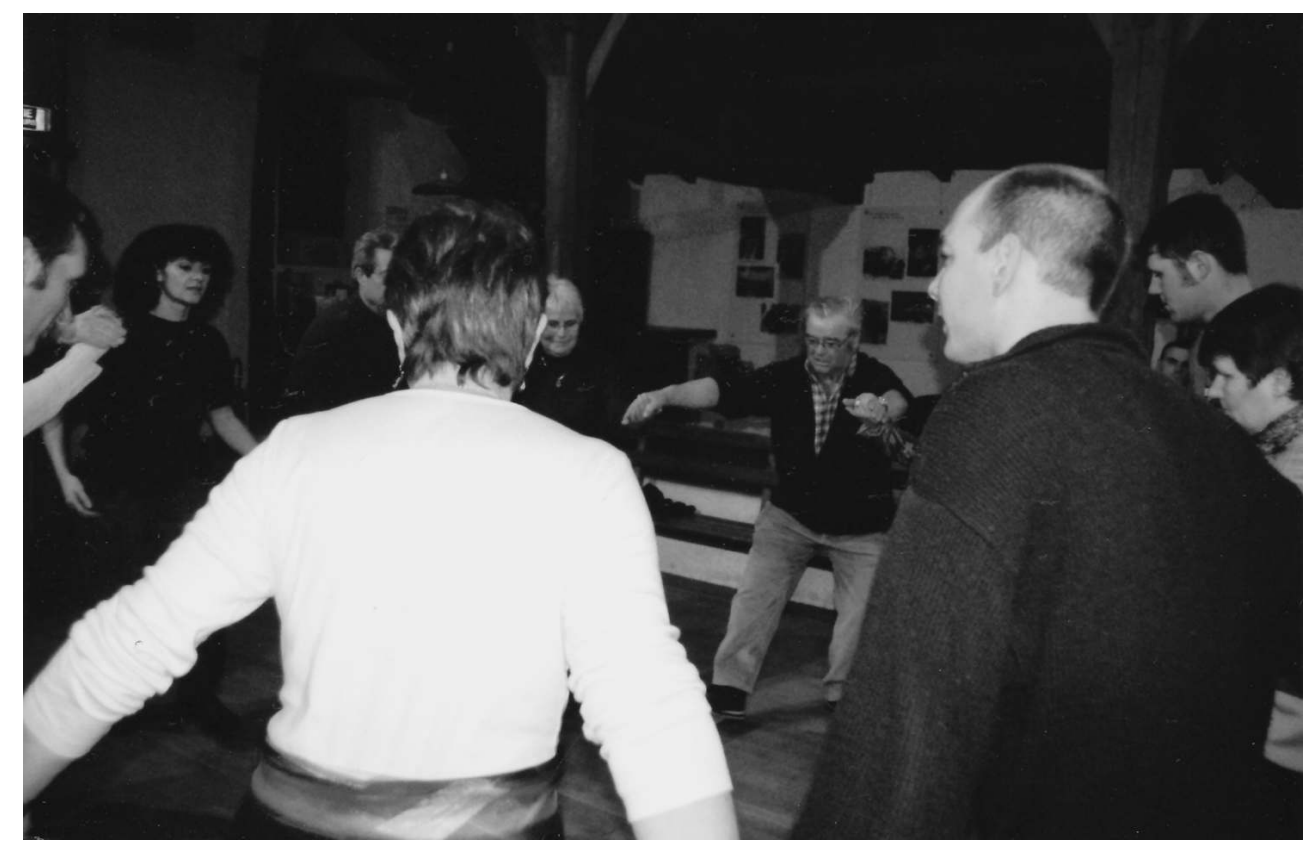

On pourrait, bien que la question de l'improvisation poétique chantée paraisse avoir singulièrement peu intéressé la plupart des auteurs, multiplier ces témoignages à l'envi. Gageons d'ailleurs que bien des bouches inconnues pourraient nous être, dans cette tâche, d'un aussi grand secours que tout le fonds de la B.N.F. Et, si cela ne suffisait pas, je pourrais ajouter que j'ai moi-même entendu il y a peu, autour d'une table vendéenne, de tels couplets improvisés pour prolonger de façon humoristique quelques chansons traditionnelles ${ }^{35}$. 
65 En second lieu, nous pouvons invoquer un imparable argument de nécessité. Il est bien évident qu'en contexte fonctionnel, par exemple au cours d'un bal mené à la voix (« à la gueule») comme il s'en donnait tant en France il y a encore moins d'un siècle, il était indispensable que les chanteurs, qui avaient aussi un rôle de "meneurs», puissent improviser des paroles au pied levé. Pas question d'hésiter, de «tralaliser» sans fin ni d'arrêter une danse sous prétexte d'un trou de mémoire ou du fait que la chanson se trouve terminée...

66 Enfin, il convient d'apprécier l'agrément que devait apporter aux chanteurs, dans le contexte répétitif de la chanson fonctionnelle, l'ouverture à une pratique improvisatoire, de même qu'aucun instrumentiste «trad» actuel ne conçoit son répertoire fonctionnel (bals folks...) sans une part de variations et d'improvisation. Or, dans la chanson française où le chant est essentiellement syllabique et où, donc, l'improvisation vocale mélodique est très restreinte, l'improvisation poétique paraît à peu près la seule qui permette à l'imagination du chanteur de s'exprimer. Elle constitue donc, d'un point de vue mental et ludique, un précieux espace de liberté.

\section{L'improvisation: une source de tradition?}

Cette réflexion a voulu montrer qu'une forme poético-musicale, en révélant une nature propice à l'IPC, peut constituer un indice du passé improvisatoire ou semi-improvisatoire d'un genre vocal au répertoire aujourd'hui en grande partie fixé; comme si la forme fossilisait, en quelque sorte, la pratique dont elle est née.

68 Sur un autre plan, il est possible que l'hypothèse avancée ici rejoigne la question du sens de la «chanson traditionnelle». Si l'on pense que la chanson a été longtemps l'objet de pratiques improvisatoires ou semi-improvisatoires, pratiques par nature individuelles et éphémères, de quelle manière et selon quels critères a-t-elle pu se transmettre de génération en génération?

69 Il est probable que ce que nous concevons aujourd'hui plus ou moins comme un répertoire d'« œuvres» traditionnelles ait été, par le passé, davantage conçu comme un répertoire de formes, de structures poético-musicales sur lesquelles pouvait se poser un texte connu ou nouveau, un texte varié, voire en partie improvisé. Les feuilles volantes nous laissent un témoignage de cette conception, en indiquant que telles nouvelles paroles doivent se chanter sur tel air, tel timbre rappelé par le titre de sa chanson originelle, mais qui a de fait acquis son autonomie par rapport à cette " chanson»; car ce titre n'évoque plus une œuvre précise mais un timbre, qui n'est rien d'autre qu'une structure poético-musicale destinée à recevoir des strophes adéquates.

70 Et ces strophes, pour rester ouvertes à une réinterprétation improvisatoire, doivent ellesmêmes être conçues non comme un texte définitif, mais plutôt comme un canevas, dont certaines parties seront susceptibles d'être variées à l'infini. On peut alors considérer que ce que nous appelons «chanson traditionnelle» a pu être longtemps pensé comme un genre "à canevas»" ${ }^{36}$, ce qui nous ramène à d'autres domaines d'improvisation, où le canevas peut être explicite (cf. la commedia dell'arte, le jazz, etc.) ou implicite (cf. les joutes oratoires et autres genres d'IPC « pure», l'art oratoire, etc.). La chanson apparaît dès lors comme un moule, une forme-système qui développe sa matière d'après un canevas préétabli, souvent de nature narrative - dans les répertoires les plus visiblement ouverts à l'improvisation, notamment les chansons en laisse -, ou séquentielle ( $c f$. La laine des 
moutons...). Ce canevas, cette structure nodulaire minimale ${ }^{37}$, probablement issu du modèle originel de la chanson, se dégage souvent des multiples versions qui en sont collectées, versions dont il constitue une sorte d'invariant sémantique stable, comme ceci a été maintes fois relevé. Mais la multiplicité, voire l'indénombrabilité de ces «variantes», que l'on attribue souvent essentiellement à une transmission fautive, ne pourrait-elle être aussi le fruit de ces «variations volontaires» ${ }^{38}$, plaidant par là-même pour une conception structurelle de la chanson, ouverte à des pratiques de variation et d'improvisation? Cette improvisation qu'il est si tentant de développer ${ }^{39}$, comme en témoignent généralement les versions relevées, sur les « détails» - hors trame minimale d'un texte de chanson: où le galant s'est caché, ce que les rats en ont mangé, en quels objets de piété il a été recyclé, etc. La vocation du canevas n'est-elle pas de servir à «broder»?

Il y a donc, dans cette acception de la chanson «traditionnelle», une part de transmission de savoir-faire (en particulier, de savoir-improviser) tout aussi importante que celle du savoir et $\mathrm{du}$ répertoire. Et là réside peut-être une clef de l'aptitude à la «traditionnalisation» d'une forme poético-musicale: c'est peut-être cette faculté de «se laisser improviser», combinée à une certaine attractivité du canevas, qui, passé le succès populaire initial, va permettre à la chanson de se diffuser oralement, de traverser le temps et de prendre une dimension traditionnelle. Quand on commence à oublier les paroles du Poinçonneur des Lilas, on n'ose plus le chanter; quand on oublie celles du Galant oublié dans l'armoire, on peut bien les réinventer, ce qui procure en outre un délicieux amusement.

Finalement, il pourrait en aller de la chanson comme de toute matière vivante: peut-être est-ce cette capacité de la forme à se laisser déformer qui permet sa conservation dans le temps.

Lous partajou, lous partajou,Crompou de moucodous,Lous partajou, ni fòu dous.(Les filles de Saint-Chély achètent des mouchoirs; elles les partagent: d'un elles font deux)On peut également imaginer les improvisations auxquelles peuvent donner lieu des chants comme ceux qui accompagnent les « rondes de cocus», dansées en temps de carnaval.

\section{BIBLIOGRAPHIE}

AYMÉ Marcel, 1929, La Table-aux-Crevés. Paris: Gallimard.

BARTHES Roland, 1966, « Introduction à l'analyse structurale du récit». Communications 8. Paris.

BEC Pierre, 1999, « Le vers long à césure dans les débats chantés traditionnels». À la croisée des chemins. Musiques savantes-Musiques populaires. St-Jouin-de-Milly: FAMDT Éditions.

CERVANTES Miguel de, 2002, Novelas ejemplares (La ilustre fregona), tome II. Madrid: Ediciones Cátedra. 
CORNULIER Benoît de, 1996, «Le folklore refoulé et travesti dans la poésie française». Repression and Expression, Literary and Social Coding in Nineteenth-Century France. New York: Peter Lang.

CRIVILLÉ I BARGALLÓ Josep, 1988, Historia de la Música española, El Folklore musical. Madrid: Alianza Música (1 ${ }^{\text {re }}$ édition 1983).

DE CROZE Austin, 1976, La Chanson populaire de l'Ile de Corse. Marseille: Laffitte Reprints (d'après Paris, 1911).

DE ZERBI Ghjermana, 1981, Cantu Nustrale. Bastia: Scola Corsa, Accademia d'I Vagabondi.

GUILCHER Jean-Michel, 1997, La Tradition populaire de danse en Basse-Bretagne. Spézet-Douarnenez: Coop. Breizh-Chasse-Marée/Armen (1 ${ }^{\text {ère }}$ édition 1963).

LAFORTE Conrad, 1981, Survivances médiévales dans la chanson folklorique. Poétique de la chanson en laisse. Sainte-Foy (Québec): Presses de l'Université Laval.

LAFORTE Conrad, 1993, Poétiques de la chanson traditionnelle française. Sainte-Foy (Québec): Presses de l'Université Laval (1è édition 1976).

LAFORTE Conrad, 1997, Chansons de facture médiévale retrouvées dans la tradition orale. Montréal: Nuit Blanche.

LAMBERT Louis, 1983, Chants et Chansons populaires du Languedoc. Marseille: Laffitte Reprints (d'après Paris/Leipzig, 1906)

MANCA Maria, 2002, La Poésie pour répondre au Hasard, Une approche anthropologique des joutes poétiques en Sardaigne. Thèse de doctorat: Université Paris X.

NODA GÓMEZ Talio, 1998, La Música tradicional canaria, hoy. Las Palmas: Fundación Orquesta Filarmónica de Gran Canaria.

RIVIÈRE Hervé, 1991, « Sur une structure poético-musicale». Poésies chantées de tradition orale en Flandre et en Bretagne. Paris: Champion.

SOUTHWELL-COLUCCI Edith, 1996, Chants populaires corses. Borgu: Mediterranéa (d'après Canti populari corsi, Livourne, 1933), traduction de Sauveur Soddu.

\section{Référence discographique}

1997, Los Campesinos (Lanzarote): Isla con viento. Tenerife: Centro de la Cultura Popular Canaria, CD-337.

\section{NOTES}

1. Il s'agit d'une suite de coplas (quatrains d'octosyllabes assonancés aux vers pairs: xAyA', $\mathrm{xByB}$ '..., parfois considérés comme distiques de vers longs $8+8$ ), strophes utilisées par excellence comme leurs ancêtres et homonymes romanes médiévales - dans la poésie improvisée hispanique, tant narrative que lyrique; le texte dit en substance: «Que s'avance la belle Argüello, jeune fille, à peine, et qu'en faisant une révérence elle retourne deux pas en arrière. Que la ramène par la main celui qu'on appelle Barrabas, muletier andalou, chanoine du Compas. Des deux servantes galiciennes qui sont dans cette auberge, que s'avance maintenant la plus joufflue, en bras de chemise et sans tablier. Que Torote l'empoigne et que tous les quatre, avec des gesticulations et des déhanchements, entament un contrapás.» 
2. Si j'excepte le cas des tavernes flamencas où l'on est souvent friand de siguiriyas, ces dernières n'ayant d'ailleurs, le nom mis à part, à peu près rien de commun avec la seguidilla.

3. Petit cistre à doubles cordes métalliques, utilisé comme voix instrumentale supérieure dans un rôle de conduite et d'impulsion mélodico-rythmique.

4. Ce qui correspond, rappelons-le, à un quatrain irrégulier $(7+5+7+5)$ assonancé aux vers pairs (xAyA'), soit encore à un distique de dodécasyllabes assonancés, césurés après la septième syllabe.

5. Premier vers dont une règle implicite d'invention me semble être qu'il doit si possible réaliser, outre une certaine continuité sémantique, une assonance approximative (parfois très approximative, certes) avec le dernier vers de la strophe précédente: salto/bailando, pantorrillas/ bailas, bonita/cintura, cualquiera/guitarra.

6. Littéralement: «Tu me demandes des seguidillas (couplets), je te les chante; saisis-les au vol, en faisant un saut/Quand tu sautes en dansant les seguidillas, tous gardent les yeux rivés à tes jambes/Chaque fois que tu danses, mon cœur se cabre dans ma poitrine, belle enfant/Ta taille et tes hanches ravissent quiconque te voit danser/Le timple et la guitare vont de concert pour jouer les seguidillas, celles que tu danses.»

7. Exception faite, toutefois, du cas particulier des « seguidillas» cubaines, qui n'ont rien à voir avec les seguidillas traditionnelles canariennes ou espagnoles (décidément...) puisque, se fondant sur l'étymologie directe du mot (seguir = suivre), elles consistent, pour un seul chanteur et sans aucun interlude instrumental, à enchaîner entre elles une série de décimas (dizains d'octosyllabes ABBA AC CDDC) devant un public, en y introduisant des commentaires sur le contexte, les personnalités présentes, etc. Genre qui constitue donc une pure démonstration de virtuosité improvisatoire, réservée à quelques rares chanteurs prodiges.

8. Il serait d'ailleurs certainement tout aussi intéressant de la développer sur des formes dont on sent bien qu'elles ne « marcheraient» pas. Je laisse ainsi tout loisir au lecteur de prouver qu'il est bien malaisé d'improviser, par exemple, sur la forme poético-musicale du Poinçonneur des Lilas ou de l'Air des Bijoux.

9. C'est-à-dire emmêlant deux sujets, chantés par un seul, à la différence du chant alterné, au sujet unique, chanté par deux ou plusieurs improvisateurs.

10. Le genre dont il est ici question est celui du lamentu.

11. L'auteur évoque ici la forme de la strophe qui sert principalement à l'improvisation corse, à savoir le sizain d'octosyllabes assonancés aux vers pairs, xAyA'zA ", que l'on peut aussi considérer comme un tercet (terzinu) de vers longs assonancés et fortement cesurés. Cette strophe s'emploie notamment dans le chjama è respondi, mais peut aussi être utilisée dans les nanne, lamenti, serenati, canzone d'elezzioni, voceri...

12. Dolu: cérémonie funèbre; tola: table où l'on expose un mort.

13. Dont on peut relever que les Corses l'appellent bien "terzinu» (tercet), et non "sesta» (sizain); à ce sujet, cf. Bec (1999: 174-188). De même que, comme nous le rappelle Conrad Laforte, la copla, quoique souvent transcrite sous forme de quatrain, tire vraisemblablement, comme la cobla et le couplet, son étymologie du « couple» de vers longs (Laforte 1993: 38).

On peut par ailleurs remarquer que la structure poétique du terzinu se voit la plupart du temps renforcée par l'organisation mélodico-harmonique du chant: ainsi, les octosyllabes impairs se terminent le plus souvent sur un degré suspensif du mode (en général II, III ou V), tandis que l'assonance des octosyllabes pairs est soulignée par un fort appui sur la finale du mode.

14. Comme le montre par exemple, dans sa thèse, Maria Manca à propos de la gara poetica sarde (2002: 160 et suiv.).

15. Il est en effet intéressant de constater la présence extrêmement répandue de ces deux types de profil mélodique dans les manifestations les plus diverses d'IPC, que j'ai pu relever notamment dans les sphères latines et arabes. Dans les joutes, la forme mélodique «en dents de scie» (typiquement, un saut ascendant initial pouvant aller d'une quinte à une octave, suivi d'une 
longue phrase descendante conjointe et oscillante sur les degrés de l'échelle modale) prédomine nettement, à la fois pour des raisons pratiques (nécessité, pour celui qui prend la parole, d'attirer l'attention des auditeurs ou de couvrir la voix de l'autre), symboliques (représentation de l'énergie du souffle ou de l'inspiration poétique, lien avec la structure de la strophe), ou encore métaphoriques (par exemple, analogie du chant du poète avec le chant du coq, symbole de vaillance au combat et de virilité...).

16. i.e. permettant une certaine souplesse entre mémoire et improvisation.

17. On peut d'ailleurs relever bien d'autres irrégularités - syntaxiques, métriques, etc. - dans les recueils de chansons traditionnelles, qui laissent penser que l'improvisateur potentiel n'y est guère inhibé par une exigence de perfection formelle. Conrad Laforte, sans aborder le sujet de l'improvisation à proprement parler, évoque celui des "accommodements improvisés»: "Ces chansons en laisse étant de tradition orale, il arrive que le chanteur soit obligé de suppléer à l'oubli d'un mot ou d'une expression par des accommodements improvisés. Il n'hésitera pas à remplacer un terme qu'il a oublié et, si le paradigme qui se présente à son esprit est plus court, il double une syllabe ou ajoute une muette. Si le mot est plus long, il fait une élision ou, par une sorte d'acrobatie, il divise une note pour chanter deux syllabes en augmentant le tempo, car l'accent tonique des paroles doit arriver en même temps que celui de la mélodie. Cette technique est propice à une certaine traduction orale et aussi à l'utilisation du jeu très important des muettes qui apportent une sorte d'élasticité du fait qu'elles peuvent être prononcées ou élidées ou même ajoutées» (Laforte 1981: 24).

18. Ce qui fait, soit dit en passant, que l'oralissime vers de laisse peut très bien correspondre à un alexandrin. On pourrait par exemple, en tout état de cause, construire une strophe du Galant sur «Un seul être vous manque / et tout est dépeuplé» (diguedondadondaine), bien que le rapport direct ne saute pas aux yeux.

19. A ce sujet, j'ai pu observer que les improvisateurs - parfois analphabètes - préféraient souvent, à la vision « en tableau» que nous nous en faisons volontiers (de type: ABBAACCDDC...), un ensemble de recettes locales établissant une stratégie mémorielle fondée sur le comptage: le quatrième vers doit rimer avec le premier (d'où mémorisation particulière de la première rime), le dixième avec le sixième, etc. (et d'où le fait, « digital», que je n'aie jamais observé de strophes improvisées de plus de dix vers?).

20. Où a est presque toujours masculine, et $b$ féminine.

21. Cet exemple illustre par ailleurs que le deuxième vers du rabé-raa, parfois considéré comme «blanc», rime très souvent avec le deuxième vers des strophes suivantes - qui, parfois, ne fait que le répéter -, formant ainsi une chaîne rimique secondaire sur l'ensemble de la chanson.

22. En fait, on pourrait aussi parler d'attente/résolution, puisque le rabé-raa apparaît bien souvent comme un distique amputé ou varié, suivi de sa version « vraie» et définitive.

23. Avec, typiquement, une terminaison mélodique sur le deuxième, le troisième ou le cinquième degré, ce que l'on retrouve dans le kan ha diskan breton, le chjama è respondi corse, le punto cubain et bien d'autres genres soumis à l'IPC, aux points clefs (fins de certains vers ou hémistiches) de l'improvisation.

24. De nombreuses formes, dans les genres poétiques improvisés, jouent sur cet effet de «surprise» finale attendue et plus ou moins différée. J'en ai relevé plusieurs, par exemple, dans les genres d'IPC à Cuba. Mais on pourrait en trouver dans bien d'autres domaines ouverts à l'improvisation et notamment à l'improvisation comique, de la commedia dell'arte à l'art du clown.

25. $C f$. encore une fois l'exemple 3 bis, qui joue sur une pratique particulière de «tralalisation» des mots pour engendrer du temps d'énonciation. Bien sûr, ce principe de " gain de temps» n'est pas une preuve absolue d'un caractère improvisatoire. En effet, il peut aussi bien s'appliquer à une fabrication «hors-temps» (aurait dit Xenakis), écrite ou pensée, de la chanson, dans la mesure où il est simplement destiné à faciliter l'invention, qu'elle soit instantanée ou différée. Il correspond en cela au principe général, essentiel dans le cadre des chansons fonctionnelles, qui 
consiste à créer un maximum de temps musical avec un minimum de matière littéraire, puisqu'il s'agit de «nourrir» musicalement un temps long, étiré, comme celui de la marche, de la danse ou du travail. L'on voit bien, par exemple, l'application de ce principe dans les chansons de type énumératif, telles que les chansons de "dizaines» (destinées en particulier à accompagner les longues marches dans les provinces de l'Ouest), lesquelles, étant régies par un processus automatique, ne sont nullement improvisées. D'où l'intérêt essentiel d'examiner dans une forme poético-musicale, non seulement le rapport quantité de texte/temps d'énonciation, mais surtout la gestion de ce temps créé, et son niveau d'adéquation à une conduite improvisatoire.

26. Du fait que le temps de préparation silencieux est bien plus efficace que celui durant lequel l'improvisateur doit continuer à chanter tout en réfléchissant; paramètre qu'il est difficile de mesurer quantitativement.

27. Ici, pas de thermodynamique qui tienne... Il faut essayer d'improviser soi-même pour se rendre compte de ces aspects qualitatifs déterminants.

28. Cf. les soixante-treize formules strophiques répertoriées, analysées et classifiées pour la chanson en laisse dans (Laforte 1981).

29. Elément qui se trouve, je crois, dans tous les genres ouverts à l'IPC. Dans la "musique paysanne» cubaine, par exemple, chaque chanteur se constitue un réservoir de strophes (décimas, redondillas, "estribillos» d'une forme particulière, etc.) et un réservoir de "tonadas» (profils mélodiques) adaptés à la forme de ces strophes, dans lesquels il puise selon les circonstances pour assembler paroles et musique en une forme poético-musicale sans cesse renouvelée. En Bretagne intérieure, autre terre notable de poètes improvisateurs (cf le kan a boz improvisé en temps non mesuré, de même que les strophes de compliment, poz-kan, les joutes poétiques du diskour [philosophique] et du prokont [grivois], la coutume du grassoù, psalmodie funèbre souvent improvisée par des femmes, ou encore celle d'improviser quelques paroles lorsqu'on «note» ou « gavotte» en pays gallo, i.e. qu'on accompagne la danse par des couplets chantés mêlés de tralalas), cette même caractéristique se retrouve par exemple dans la chanson fonctionnelle, comme le rappelle Jean-Michel Guilcher à propos du kan ha diskan: «Pour saisir le déroulement d'une gavotte chantée en Haute-Cornouaille, il faut savoir que la chanson employée à cet usage n'est pas un assemblage fixe de musique et de paroles. Il existe d'une part un répertoire de poèmes, d'autre part un répertoire d'airs, les «tons». Poèmes et «tons», établis sur des modèles traditionnels, sont adaptables les uns aux autres. Un chanteur choisit le texte qu'il veut dire, et l'air sur lequel il va le chanter» (Guilcher 1997: 242-243).

30. Exactement comme, dans le domaine du théâtre ou du pré-théâtre improvisé, on peut en trouver par exemple dans le théâtre antique ou la commedia dell'arte, dans les joutes poétiques, dans l'art oratoire, etc.

31. Cf. Guilcher (1997: 244-251), qui cite par exemple: « N'oun ket ken evid kana gand ar sehed am eus / Kar va laiñchenn 'zo frailhet, ha va zeod 'za (d)a dreuz.» (Je ne peux plus chanter, tant j'ai soif car mon gosier est crevassé et ma langue va de travers).

32. Me donneriez-vous, Bonne Marie, quelque chose qui fasse bien mourir ceux qui veulent toujours du mal aux hommes dont le mien est le plus beau [...], in Aymé (1929: 208-209).

33. Exemple: Lai filhos de Sent-Chélis / Crompou de moucodous, (bis)

34. Photographie de J.-P. Bertrand (fonds EthnoDoc-Arexcpo, mars 2003); le meneur est Michel Raballand, ici vu de face. Le «boulité» ou "tralala» est, dans le Marais Breton vendéen, l'épisode de la ronde pendant lequel le meneur improvise des tralalas, des phrases sans queue ni tête ou des allusions contextuelles (en dialecte maraîchin), dans le principal but d'amener les danseurs à se tromper lors de changements de pas délicats.

35. Oserai-je d'ailleurs avouer que, la chose étant tellement aisée et naturelle, je me suis moimême risquée à improviser quelques vers? Ce que, en outre, mes informateurs cubains s'amusent souvent à me faire faire... en espagnol. 
36. Patrice Coirault écrivait que la poésie populaire de tradition orale est « plus un sens, une idée directrice [...] qu'un assemblage fixe de mots choisis»; cité par Guilcher (1997: 269).

37. Cf. Barthes (1966: 1-27), qui compare le récit à une longue phrase structurée autour d'un énoncé minimal fonctionnel.

38. Selon l'expression de Jeanine Fribourg.

39. De même que l'on enrichit tout en parlant une phrase de divers compléments circonstanciels et incises; de même que les bouffons italiens agrémentaient "à l'impromptu» leurs pièces improvisées de lazzi variés, etc.

\section{RÉSUMÉS}

L'examen de différents répertoires traditionnels qui servent actuellement, dans le monde latin, de support à des improvisations poétiques chantées (IPC), permet de dégager certaines constantes formelles qui semblent elles-mêmes des implications directes du processus d'improvisation, notamment lorsque celle-ci doit se dérouler dans un temps rigide et imposé par exemple, dans le cadre de répertoires à fonction et à contraintes précises dans le temps, comme les répertoires à danser.

Or il est troublant de constater la présence, dans des catégories de chanson traditionnelle dont le contenu littéraire apparaît aujourd'hui comme relativement figé et non sujet à l'improvisation, de certains de ces mêmes aspects formels, agencés suivant une stratégie temporelle proche de celle des genres improvisés.

Serait-il alors audacieux d'avancer l'hypothèse que certaines de ces catégories de chansons ont $\mathrm{pu}$, en un temps passé plus ou moins reculé, servir elles-mêmes de support à des improvisations poétiques chantées, et retirer l'essentiel de leurs caractéristiques formelles de cette ancienne pratique improvisatoire?

\section{AUTEUR}

\section{MARIE-HORTENSE LACROIX}

Marie-Hortense LACROIX est née en 1972. A l'issue d'études scientifiques (ingénieur diplômée d'études approfondies en sciences de la matière), elle se consacre à la musique, à l'ethnomusicologie, à la musicologie et à l'écriture littéraire. Actuellement enseignante de musicologie à l'université Paris IV, elle écrit une thèse sur l'improvisation poétique chantée dans le monde latin et en particulier à Cuba (province de Sancti Spíritus), sous la direction de François picard. 